\title{
A Comparasion of Laboratory and Cardiopulmonary Effects of Desflurane, Detomidine and Medetomidine Anaesthetic Combinations in Horses
}

\author{
Hanifi Erol' \& Mustafa Arıcan²
}

\begin{abstract}
Background: Equine anesthesia morbidity and mortality rates are greater than in other domestic animals because of hypotension and hypoventilation. The important features desired in general anesthesia for horses are a rapid effect, rapid emergence and balanced anesthesia. The long duration of action of currently used anesthetic agents cause various complications in horses. The aim of the present study was to compare the clinical effects of combination of the anesthetics desflurane, detomidine and medetomidine in horses.

Materials, Methods \& Results: Eight healthy mixed-breed horses (four males and four females) with weighing $275 \pm 56 \mathrm{~kg}$ [mean \pm standard deviation $(\mathrm{SD})]$ and aged $6.8 \pm 5$ years $[(\operatorname{mean} \pm \mathrm{SD})]$ were used for this study. The horses were placed into one of four groups: group I (detomidine-desflurane), group II (detomidine-desflurane-atipamezole), group III (medetomidinedesflurane), or group IV (medetomidine-desflurane-atipamezole). Horses were rested for 15 days before each group starts to study. Intravenous detomidine $(25 \mu \mathrm{g} / \mathrm{kg})$ was used for premedication in groups I and II, and intravenous medetomidine $(7 \mu \mathrm{g} /$ $\mathrm{kg})$ was used for premedication in groups III and IV. Ketamine hydrocholoride $(2 \mathrm{mg} / \mathrm{kg})$ and midazolam $(0.03 \mathrm{mg} / \mathrm{kg}) \mathrm{were}$ intravenously administered in the same syringe to induce anesthesia. After induction of anesthesia, horses were placed in the left lateral recumbent position, and the trachea was intubated with a cuffed endotracheal tube with an internal diameter of $28 \mathrm{~mm}$. The endotracheal tube was attached to a large animal circle breathing system anesthesia machine, and anesthesia was maintained with desflurane for $90 \mathrm{~min}$. The initial dosage of desflurane was $14 \%+4 \mathrm{~L} \mathrm{O}_{2} / \mathrm{min}$, and was reduced by $2 \%$ every 10 min over the first $30 \mathrm{~min}$ of anesthesia. After $30 \mathrm{~min}$, the desflurane dose was changed to $8 \%+4 \mathrm{~L}$, which was maintained until the end of anesthesia (90 min). After $90 \mathrm{~min}$, the administration of desflurane was discontinued, and all animals were supported by $\mathrm{O}_{2}$, with groups II and IV receiving $0.06 \mathrm{mg} / \mathrm{kg}$ atipamezole in addition to oxygen. Anaesthetic action times, hematological parameters, blood gas levels, electrolyte levels, biochemical values, electrocardiography values and end-tidal carbon dioxide volume were measured before, during, at the end of, and $24 \mathrm{~h}$ after anesthesia.

Discussion: In this study, medetomidine $(7 \mu \mathrm{g} / \mathrm{kg})$ and detomidine $(25 \mu \mathrm{g} / \mathrm{kg})$ were intravenously administered, which was adequate and suitable for sedating horses. At the end of anesthesia, $0.06 \mathrm{mg} / \mathrm{kg}$ atipamezole was intravenously administered in groups II and IV. However, atipamezole did not affect the clinical parameters. Stress, excitement, fear, catecholamine exchange in blood circulation, hyperglycemia, and hypoxia can all cause changes in venous blood parameters. These are potential reasons for the changes in venous blood parameters (i.e., WBC and $\mathrm{Hb}$ ) observed at the beginning of and during anesthesia in the present study. During and after the anesthetic period, serum biochemical values can be different from baseline values. They are dependent on the effects of anesthetic agents. During anesthesia, the decrease and increase of biochemical values stabilize the changes in the enzyme system that develops because of the effects of anesthetic agents. In the present study, it was considered that the changes in the biochemical values aimed to stabilize the changes induced by anesthesia. Regarding the electrolyte parameters evaluated in the study, there was a statistical difference detected in Na values between $90 \mathrm{~min}$ after induction of anesthesia and $24 \mathrm{~h}$ after induction of anesthesia in group IV. However, in previous studies, the changes in $\mathrm{Na}$ values did not influence the cardiac pressure during general anesthesia. In our study, significant changes were not seen in any electrolyte parameters except $\mathrm{Na}$, and atrioventricular block was not detected in ECG traces. Generally, decreased $\mathrm{ETCO}_{2}$ levels are evidence of lung perfusion deficiency. It depends on the effects of anesthetic agents on the cardiopulmonary, cardiovascular, and respiratory systems. In particular, the higher pressure and dose of desflurane supress respiratory system. Oxygen supplementation in general anesthesia increases respiratory rate, but $a-2$ agonists and ketamine-midazolam effects can eliminate the increasing respiratory rate in general anesthesia.
\end{abstract}

Keywords: anesthesia, horse, desflurane. 


\section{INTRODUCTION}

Equine anesthesia morbidity and mortality rates are greater than in other domestic animals because of hypotension and hypoventilation [12]. Detomidine is a specific alpha_2 ( $a$-2) adrenoreceptor agonist commonly used in horses [7,22]. The sedative and analgesic effects of detomidine depend on its binding to $a-2$ receptors in the locus coeruleus complex, brain stem and spinal cord [6]. Medetomidine is formed by the combination of the two optical enontiomers dexmedetomidine and levomedetomidine. It is a more potent and selective a-2 adrenoreceptor agonist than xylazine or detomidine [2,5,29]. Benzodiazepines are the most frequently used psychotropic drugs in animals. They act on gamma-aminobutyric acid (GABA) receptors located in the central nervous system $[13,14,16]$. Benzodiazepines are rarely used alone in horses because they cause muscle weakness and ataxia [12,30]. Atipamezole is a potent and selective a-2 receptor blocking agent that enhances noradrenaline release in the central and peripheral nervous system. It inhibits or eliminates the effects of the $a$-2 adrenoceptor agonists detomidine and medetomidine. $[4,11,18]$. Desflurane has the lowest blood-gas partition coefficient (0.42) and the highest minimum alveolar concentration (MAC) value (7.2 in horses) among inhaled anesthestic agents. It provides rapid induction of anesthesia, as well as rapid emergence [23]. Only a small proportion of desflurane is metabolized in the body and it does not cause major toxic effect in the liver or urinary system [3]. The aim of the present study was to compare the clinical effects of combination of the anesthetics desflurane, detomidine and medetomidine in horses.

\section{MATERIALS AND METHODS}

\section{Animals}

Eight healthy mixed-breed horses (four males and four females) with weighing $275 \pm 56 \mathrm{~kg}$ [mean \pm standard deviation (SD)] and aged $6.8 \pm 5$ years [(mean $\pm \mathrm{SD}$ )] were used for this study. The clinical examination and hematological tests were done before starting the study. The horses were placed into one of four groups: group I (detomidine-desflurane), group II (detomidinedesflurane-atipamezole), group III (medetomidinedesflurane), or group IV (medetomidine-desfluraneatipamezole). Horses were rested for 15 days before each group starts to study. All horses were fasted for 12 $\mathrm{h}$ before premedication; no restriction was applied on water intake. Before premedication, the body weight, respiratory rate, body temperature, and heart rate of horses were recorded in examination papers.

\section{Clinical evaluation and anesthesia}

Before premedication, a 20-gauge intravenous catheter was placed in the right jugular vein for blood sample collection and administration of anesthetic drugs. Electrocardiography (ECG) readings, oxygen saturation $\left(\mathrm{SpO}_{2} \%\right)$, and extremity derivations (aVL, aVR, aVF) were measured by a BM3 Vet monitor ${ }^{1}$ before premedication and during anesthesia. End-tidal carbon dioxide volume $\left(\mathrm{EtCO}_{2}\right)$ was measured (Medair $82450)^{2}$ during anaesthesia. Intravenous detomidine ( $25 \mu \mathrm{g} / \mathrm{kg}$, Domosedan $)^{3}$ was used for premedication in groups I and II, and intravenous medetomidine (7 $\mu \mathrm{g} / \mathrm{kg}$, Domitor $)^{3}$ was used for premedication in groups III and IV. Ketamine hydrocholoride $(2 \mathrm{mg} / \mathrm{kg}$, Ketasol $10 \%)^{4}$ and midazolam $(0.03 \mathrm{mg} / \mathrm{kg} \text {, Demizolam })^{5}$ were intravenously administered in the same syringe to induce anesthesia. After induction of anesthesia, horses were placed in the left lateral recumbent position, and the trachea was intubated with a cuffed endotracheal tube with an internal diameter of $28 \mathrm{~mm}$. The endotracheal tube was attached to a large animal circle breathing system anesthesia machine (Large animal LSD 3000 anesthetic machine $)^{6}$, and anesthesia was maintained with desflurane (Suprane) ${ }^{7}$ for $90 \mathrm{~min}$. The initial dosage of desflurane was $14 \%+4 \mathrm{~L} \mathrm{O}_{2} /$ $\mathrm{min}$, and was reduced by $2 \%$ every $10 \mathrm{~min}$ over the first $30 \mathrm{~min}$ of anesthesia. After $30 \mathrm{~min}$, the desflurane dose was changed to $8 \%+4 \mathrm{~L}$, which was maintained until the end of anesthesia (90 min). After $90 \mathrm{~min}$, the administration of desflurane was discontinued, and all animals were supported by $\mathrm{O}_{2}$, with groups II and IV receiving $0.06 \mathrm{mg} / \mathrm{kg}$ atipamezole (Antisedan) ${ }^{3}$ in addition to oxygen.

After the anesthesia wore off and the palpebral reflex loss and returned, the spontaneous respiration, extubation, sternoabdominal position and standing up times were recorded (Table 1). ECG results, blood gas levels (GEM premier 3000) ${ }^{8}$, and the white blood count (WBC), blood $\mathrm{pH}$, venous partial carbon dioxide $\left(\mathrm{PCO}_{2}\right)$, venous partial oxygen pressure $\left(\mathrm{PO}_{2}\right)$, total carbon dioxide $\left(\mathrm{tCO}_{2}\right)$, hemoglobin level $(\mathrm{Hb})$, hematocrit $(\mathrm{Ht})$, and serum levels of bicarbonate $\left(\mathrm{HCO}_{3}\right)$, electrolytes $\left(\mathrm{Na}^{+}, \mathrm{K}^{+}, \mathrm{Ca}^{++}\right)$, lactate (Lac), and glucose 
(Glu) of venous blood (MS4e) ${ }^{9}$ were evaluated before premedication (0 $\mathrm{min}), 15,30,60$, and $90 \mathrm{~min}$ after induction of anesthesia, and $24 \mathrm{~h}$ after anesthesia (Table 2). Levels of aspartate transaminase (AST), alanine transaminase (ALT), alkaline phosphatase (ALP), creatine kinase $\mathrm{MB}(\mathrm{Ck}-\mathrm{MB})$, blood urea nitrogen (BUN), and creatine $(\mathrm{Cr})$ were evaluated before premedication, at the end of anesthesia (after $90 \mathrm{~min}$ ), and $24 \mathrm{~h}$ after anesthesia (Table 3).

\section{Data and statistical analysis}

Data were analyzed using the Shapiro-Wilk test for normality, then with a one- and two-way repeated measures ANOVA followed by Tukey or Bonferroni's significant difference tests to compare intra-group and inter-group values. Values of $P<0.05$ were considered statistically significant. All analyses were performed using IBM SPSS Statistics 21 program. Results are presented as the mean $\pm \mathrm{SD}$.

Table 1. Evaluation of anaesthetic agents actions times (mean \pm SD).

\begin{tabular}{cccccc}
\hline Group & PRLT & PRRT & ET & SAPT & SUT \\
\hline G I & $19 \pm 5^{*}$ & $20 \pm 9$ & $25 \pm 8$ & $42 \pm 15$ & $58 \pm 21$ \\
G II & $14 \pm 4$ & $21 \pm 6$ & $21 \pm 6$ & $37 \pm 14$ & $57 \pm 26$ \\
G III & $11 \pm 2^{*}$ & $21 \pm 9$ & $26 \pm 5$ & $33 \pm 9$ & $42 \pm 10$ \\
G IV & $15 \pm 4$ & $24 \pm 3$ & $24 \pm 4$ & $33 \pm 5$ & $40 \pm 8$ \\
\hline
\end{tabular}

PRLT: Palpebral Reflex Loss Time, PRRT: Palpebral Reflex Return Time, ET: Extubation Time, SAPT: Sternoabdominal Position Time, SUT: Standing Up Time.

*The significant difference between groups $(P<0.05)$.

Table 2. Haematological, blood gases and electrolyte values of groups (mean \pm SD).

\begin{tabular}{|c|c|c|c|c|c|c|c|}
\hline Value & Group & $0 \mathrm{~min}$ & $15 \mathrm{~min}$ & $30 \mathrm{~min}$ & $60 \mathrm{~min}$ & $90 \mathrm{~min}$ & $24 \mathrm{~h}$ \\
\hline \multirow{4}{*}{$\begin{array}{c}\text { WBC } \\
\left(\mathrm{mx} 10^{3} /\right. \\
\left.\mathrm{mm}^{3}\right)\end{array}$} & GI & $8 \pm 2 \mathrm{~A}, \mathrm{a}$ & $8 \pm 2 \mathrm{~A}, \mathrm{a}$ & $8 \pm 1 \mathrm{AB}, \mathrm{a}$ & $8 \pm 1 \mathrm{AB}, \mathrm{a}$ & $8 \pm 1 \mathrm{AB}, \mathrm{a}$ & $10 \pm 2 \mathrm{~A}, \mathrm{a}$ \\
\hline & GII & $8 \pm 2 \mathrm{~A}, \mathrm{a}$ & $9 \pm 1 \mathrm{~A}, \mathrm{a}$ & $9 \pm 1 \mathrm{~A}, \mathrm{a}$ & $9 \pm 2 \mathrm{~A}, \mathrm{a}$ & $8 \pm 1 \mathrm{~A}, \mathrm{a}$ & $10 \pm 2 \mathrm{~A}, \mathrm{a}$ \\
\hline & GIII & $8 \pm 1 \mathrm{~A}, \mathrm{ab}$ & $8 \pm 1 \mathrm{~A}, \mathrm{ab}$ & $7 \pm 1 \mathrm{~B}, \mathrm{ab}$ & $7 \pm 1 \mathrm{AB}, \mathrm{ab}$ & $7 \pm 1 \mathrm{~B}, \mathrm{~b}$ & $9 \pm 3 \mathrm{~A}, \mathrm{a}$ \\
\hline & GIV & $8 \pm 2 \mathrm{~A}, \mathrm{ab}$ & $8 \pm 1 \mathrm{~A}, \mathrm{ab}$ & $7 \pm 1 \mathrm{AB}, \mathrm{ab}$ & $6 \pm 2 \mathrm{~B}, \mathrm{~b}$ & $6 \pm 1 \mathrm{~B}, \mathrm{~b}$ & $9 \pm 2 \mathrm{~A}, \mathrm{a}$ \\
\hline \multirow{4}{*}{$\begin{array}{c}\mathrm{Hb} \\
(\mathrm{g} / \mathrm{dL})\end{array}$} & GI & $12 \pm 1 \mathrm{~A}, \mathrm{a}$ & $13 \pm 2 \mathrm{AB}, \mathrm{a}$ & $12 \pm 2 \mathrm{~A}, \mathrm{a}$ & $12 \pm 2 \mathrm{~A}, \mathrm{a}$ & $11 \pm 1 \mathrm{~A}, \mathrm{a}$ & $12 \pm 1 \mathrm{~A}, \mathrm{a}$ \\
\hline & GII & $11 \pm 1 \mathrm{~A}, \mathrm{a}$ & $13 \pm 2 \mathrm{~A}, \mathrm{a}$ & $13 \pm 1 \mathrm{~A}, \mathrm{a}$ & $12 \pm 1 \mathrm{~A}, \mathrm{a}$ & $12 \pm 2 \mathrm{~A}, \mathrm{a}$ & $13 \pm 1 \mathrm{~A}, \mathrm{a}$ \\
\hline & GIII & $12 \pm 1 \mathrm{~A}, \mathrm{a}$ & $11 \pm 1 \mathrm{AB}, \mathrm{a}$ & $12 \pm 2 \mathrm{~A}, \mathrm{a}$ & $11 \pm 1 \mathrm{~A}, \mathrm{a}$ & $10 \pm 1 \mathrm{~A}, \mathrm{a}$ & $12 \pm 2 \mathrm{~A}, \mathrm{a}$ \\
\hline & GIV & $11 \pm 1 \mathrm{~A}, \mathrm{a}$ & $11 \pm 1 \mathrm{~B}, \mathrm{a}$ & $11 \pm 2 \mathrm{~A}, \mathrm{a}$ & $12 \pm 4 \mathrm{~A}, \mathrm{a}$ & $10 \pm 2 \mathrm{~A}, \mathrm{a}$ & $13 \pm 2 \mathrm{~A}, \mathrm{a}$ \\
\hline \multirow{4}{*}{$\begin{array}{c}\mathrm{HCO}_{3} \\
(\mathrm{~mm} / \mathrm{L})\end{array}$} & GI & $26 \pm 2 \mathrm{~A}, \mathrm{a}$ & $25 \pm 2 \mathrm{~A}, \mathrm{a}$ & $25 \pm 3 \mathrm{~A}, \mathrm{a}$ & $24 \pm 3 \mathrm{~A}, \mathrm{a}$ & $26 \pm 3 \mathrm{~A}, \mathrm{a}$ & $27 \pm 2 \mathrm{~A}, \mathrm{a}$ \\
\hline & GII & $29 \pm 2 \mathrm{~A}, \mathrm{a}$ & $26 \pm 3 \mathrm{~A}, \mathrm{ab}$ & $25 \pm 2 \mathrm{~A}, \mathrm{~b}$ & $25 \pm 2 \mathrm{~A}, \mathrm{ab}$ & $26 \pm 3 \mathrm{~A}, \mathrm{ab}$ & $28 \pm 4 \mathrm{~A}, \mathrm{ab}$ \\
\hline & GIII & $29 \pm 2 \mathrm{~A}, \mathrm{a}$ & $26 \pm 2 \mathrm{~A}, \mathrm{a}$ & $26 \pm 2 \mathrm{~A}, \mathrm{a}$ & $27 \pm 3 \mathrm{~A}, \mathrm{a}$ & $27 \pm 4 \mathrm{~A}, \mathrm{a}$ & $29 \pm 2 \mathrm{~A}, \mathrm{a}$ \\
\hline & GIV & $30 \pm 4 \mathrm{~A}, \mathrm{a}$ & $27 \pm 4 \mathrm{~A}, \mathrm{a}$ & $26 \pm 4 \mathrm{~A}, \mathrm{a}$ & $26 \pm 4 \mathrm{~A}, \mathrm{a}$ & $27 \pm 3 \mathrm{~A}, \mathrm{a}$ & $29 \pm 2 \mathrm{~A}, \mathrm{a}$ \\
\hline \multirow{4}{*}{$\mathrm{tCO}_{2}$} & GI & $27 \pm 2 \mathrm{~A}, \mathrm{a}$ & $26 \pm 2 \mathrm{~A}, \mathrm{a}$ & $25 \pm 3 \mathrm{~A}, \mathrm{a}$ & $25 \pm 3 \mathrm{~A}, \mathrm{a}$ & $25 \pm 4 \mathrm{~A}, \mathrm{a}$ & $28 \pm 2 \mathrm{~A}, \mathrm{a}$ \\
\hline & GII & $31 \pm 2 \mathrm{~A}, \mathrm{a}$ & $27 \pm 3 \mathrm{~A}, \mathrm{ab}$ & $26 \pm 3 \mathrm{~A}, \mathrm{~b}$ & $26 \pm 2 \mathrm{~A}, \mathrm{~b}$ & $27 \pm 3 \mathrm{~A}, \mathrm{ab}$ & $29 \pm 4 \mathrm{~A}, \mathrm{ab}$ \\
\hline & GIII & $30 \pm 2 \mathrm{~A}, \mathrm{a}$ & $27 \pm 2 \mathrm{~A}, \mathrm{a}$ & $27 \pm 2 \mathrm{~A}, \mathrm{a}$ & $27 \pm 3 \mathrm{~A}, \mathrm{a}$ & $28 \pm 4 \mathrm{~A}, \mathrm{a}$ & $30 \pm 2 \mathrm{~A}, \mathrm{a}$ \\
\hline & GIV & $31 \pm 4 \mathrm{~A}, \mathrm{a}$ & $28 \pm 4 \mathrm{~A}, \mathrm{a}$ & $27 \pm 4 \mathrm{~A}, \mathrm{a}$ & $27 \pm 4 \mathrm{~A}, \mathrm{a}$ & $28 \pm 3 \mathrm{~A}, \mathrm{a}$ & $30 \pm 2 \mathrm{~A}, \mathrm{a}$ \\
\hline
\end{tabular}


(...continuation)

\begin{tabular}{|c|c|c|c|c|c|c|c|}
\hline & GI & $7,37 \pm 0,5 \mathrm{~A}, \mathrm{~b}$ & $7,40 \pm 0,5 \mathrm{~B}, \mathrm{~b}$ & $7,46 \pm 0,7 \mathrm{~B}, \mathrm{ab}$ & $7,46 \pm 0,7 \mathrm{~B}, \mathrm{ab}$ & $7,51 \pm 0,9 \mathrm{AB}, \mathrm{a}$ & $7,40 \pm 0,0 \mathrm{~A}, \mathrm{~b}$ \\
\hline \multirow{3}{*}{$\mathrm{pH}$} & GII & $7,38 \pm 0,4 \mathrm{~A}, \mathrm{bc}$ & $7,45 \pm 0,5 \mathrm{AB}, \mathrm{ab}$ & $7,48 \pm 0,6 \mathrm{AB}, \mathrm{a}$ & $7,48 \pm 0,4 \mathrm{~B}, \mathrm{a}$ & $7,50 \pm 0,5 \mathrm{AB}, \mathrm{a}$ & $7,37 \pm 0,5 \mathrm{~A}, \mathrm{c}$ \\
\hline & GIII & $7,38 \pm 0,4 \mathrm{~A}, \mathrm{bc}$ & $7,48 \pm 0,6 \mathrm{Aa}$ & $7,51 \pm 0,9 \mathrm{AB}, \mathrm{a}$ & $7,51 \pm 0,8 \mathrm{AB}, \mathrm{a}$ & $7,47 \pm 0,7 \mathrm{~B}, \mathrm{ab}$ & $7,37 \pm 0,5 \mathrm{~A}, \mathrm{c}$ \\
\hline & GIV & $7,37 \pm 0,5 \mathrm{~A}, \mathrm{c}$ & $7,51 \pm 0,3 \mathrm{~A}, \mathrm{~b}$ & $7,56 \pm 0,5 \mathrm{~A}, \mathrm{ab}$ & $7,57 \pm 0,5 \mathrm{~A}, \mathrm{a}$ & $7,57 \pm 0,5 \mathrm{~A}, \mathrm{a}$ & $7,4 \pm 0,0 \mathrm{~A}, \mathrm{c}$ \\
\hline \multirow{4}{*}{$\begin{array}{c}\mathrm{PO}_{2} \\
(\mathrm{~mm} / \mathrm{Hg})\end{array}$} & GI & $34 \pm 3 \mathrm{~A}, \mathrm{a}$ & $32 \pm 6 \mathrm{~B}, \mathrm{a}$ & $33 \pm 3 \mathrm{~A}, \mathrm{a}$ & $40 \pm 10 \mathrm{~A}, \mathrm{a}$ & $36 \pm 8 \mathrm{~A}, \mathrm{a}$ & $40 \pm 12 \mathrm{~A}, \mathrm{a}$ \\
\hline & GII & $32 \pm 7 \mathrm{~A}, \mathrm{a}$ & $33 \pm 6 \mathrm{~B}, \mathrm{a}$ & $36 \pm 6 \mathrm{~A}, \mathrm{a}$ & $41 \pm 15 \mathrm{~A}, \mathrm{a}$ & $43 \pm 13 \mathrm{~A}, \mathrm{a}$ & $44 \pm 13 \mathrm{~A}, \mathrm{a}$ \\
\hline & GIII & $33 \pm 4 \mathrm{~A}, \mathrm{a}$ & $46 \pm 12 \mathrm{AB}, \mathrm{a}$ & $68 \pm 53 \mathrm{~A}, \mathrm{a}$ & $51 \pm 22 \mathrm{~A}, \mathrm{a}$ & $46 \pm 12 \mathrm{~A}, \mathrm{a}$ & $33 \pm 5 \mathrm{~A}, \mathrm{a}$ \\
\hline & GIV & $30 \pm 3 \mathrm{~A}, \mathrm{~b}$ & $57 \pm 26 \mathrm{~A}, \mathrm{a}$ & $44 \pm 5 \mathrm{~A}, \mathrm{ab}$ & $50 \pm 11 \mathrm{~A}, \mathrm{ab}$ & $51 \pm 20 \mathrm{~A}, \mathrm{ab}$ & $33 \pm 4 \mathrm{~A}, \mathrm{~b}$ \\
\hline \multirow{4}{*}{$\begin{array}{c}\mathrm{PCO}_{2} \\
(\mathrm{mmHg})\end{array}$} & GI & $42 \pm 2 \mathrm{~A}, \mathrm{a}$ & $37 \pm 5 \mathrm{~A}, \mathrm{ab}$ & $32 \pm 5 \mathrm{~A}, \mathrm{bc}$ & $29 \pm 7 \mathrm{~A}, \mathrm{~b}$ & $28 \pm 7 \mathrm{~A}, \mathrm{~b}$ & $40 \pm 2 \mathrm{~A}, \mathrm{a}$ \\
\hline & GII & $45 \pm 3 \mathrm{~A}, \mathrm{a}$ & $35 \pm 6 \mathrm{~A}, \mathrm{~b}$ & $30 \pm 5 \mathrm{~A}, \mathrm{~b}$ & $29 \pm 3 \mathrm{~A}, \mathrm{~b}$ & $30 \pm 5 \mathrm{~A}, \mathrm{~b}$ & $43 \pm 8 \mathrm{~A}, \mathrm{a}$ \\
\hline & GIII & $45 \pm 4 \mathrm{~A}, \mathrm{a}$ & $32 \pm 5 \mathrm{~A}, \mathrm{~b}$ & $30 \pm 6 \mathrm{~A}, \mathrm{~b}$ & $30 \pm 7 \mathrm{~A}, \mathrm{~b}$ & $32 \pm 9 \mathrm{~A}, \mathrm{~b}$ & $44 \pm 3 \mathrm{~A}, \mathrm{a}$ \\
\hline & GIV & $46 \pm 5 \mathrm{~A}, \mathrm{a}$ & $30 \pm 5 \mathrm{~A}, \mathrm{~b}$ & $27 \pm 5 \mathrm{~A}, \mathrm{~b}$ & $26 \pm 5 \mathrm{~A}, \mathrm{~b}$ & $27 \pm 5 \mathrm{~A}, \mathrm{~b}$ & $44 \pm 4 \mathrm{~A}, \mathrm{a}$ \\
\hline \multirow{4}{*}{$\mathrm{O}_{2} \mathrm{SAT}$} & GI & $65 \pm 7 \mathrm{~A}, \mathrm{a}$ & $63 \pm 11 \mathrm{~B}, \mathrm{a}$ & $70 \pm 6 \mathrm{~B}, \mathrm{a}$ & $79 \pm 12 \mathrm{~A}, \mathrm{a}$ & $76 \pm 13 \mathrm{~A}, \mathrm{a}$ & $71 \pm 16 \mathrm{~A}, \mathrm{a}$ \\
\hline & GII & $61 \pm 14 \mathrm{~A}, \mathrm{a}$ & $68 \pm 11 \mathrm{~B}, \mathrm{a}$ & $76 \pm 8 \mathrm{~B}, \mathrm{a}$ & $78 \pm 18 \mathrm{~A}, \mathrm{a}$ & $81 \pm 15 \mathrm{~A}, \mathrm{a}$ & $77 \pm 12 \mathrm{~A}, \mathrm{a}$ \\
\hline & GIII & $64 \pm 8 \mathrm{~A}, \mathrm{~b}$ & $84 \pm 9 \mathrm{~A}, \mathrm{a}$ & $89 \pm 8 \mathrm{~A}, \mathrm{a}$ & $87 \pm 7 \mathrm{~A}, \mathrm{a}$ & $85 \pm 8 \mathrm{~A}, \mathrm{a}$ & $65 \pm 10 \mathrm{~A}, \mathrm{~b}$ \\
\hline & GIV & $59 \pm 5 \mathrm{~A}, \mathrm{~b}$ & $90 \pm 4 \mathrm{~A}, \mathrm{a}$ & $88 \pm 4 \mathrm{~A}, \mathrm{a}$ & $90 \pm 5 \mathrm{~A}, \mathrm{a}$ & $88 \pm 9 \mathrm{~A}, \mathrm{a}$ & $65 \pm 8 \mathrm{~A}, \mathrm{~b}$ \\
\hline \multirow{4}{*}{$\begin{array}{c}\mathrm{Lac} \\
(\mathrm{mm} / \mathrm{L})\end{array}$} & GI & $1 \pm 0,2 \mathrm{~A}, \mathrm{c}$ & $1,5 \pm 0,5 \mathrm{~A}, \mathrm{abc}$ & $2 \pm 0,6 \mathrm{~A}, \mathrm{ab}$ & $2 \pm 1 \mathrm{~A}, \mathrm{a}$ & $2 \pm 0,8 \mathrm{~A}, \mathrm{a}$ & $1 \pm 0,2 \mathrm{~A}, \mathrm{bc}$ \\
\hline & GII & $1 \pm 0,3 \mathrm{~A}, \mathrm{c}$ & $1,4 \pm 0,5 \mathrm{~A}, \mathrm{bc}$ & $2 \pm 0,4 \mathrm{~A}, \mathrm{ab}$ & $2 \pm 0,6 \mathrm{~A}, \mathrm{a}$ & $2 \pm 0,7 \mathrm{~A}, \mathrm{a}$ & $1 \pm 0,6 \mathrm{~A}, \mathrm{bc}$ \\
\hline & GIII & $1 \pm 0,2 \mathrm{~A}, \mathrm{c}$ & $1,5 \pm 0,5 \mathrm{~A}, \mathrm{ab}$ & $2 \pm 0,5 \mathrm{~A}, \mathrm{a}$ & $2 \pm 0,6 \mathrm{~A}, \mathrm{a}$ & $2 \pm 0,7 \mathrm{~A}, \mathrm{a}$ & $1 \pm 0,3 \mathrm{~A}, \mathrm{bc}$ \\
\hline & GIV & $1 \pm 0,2 \mathrm{~A}, \mathrm{c}$ & $1,2 \pm 0,3 \mathrm{~A}, \mathrm{~b}$ & $2 \pm 0,5 \mathrm{~A}, \mathrm{a}$ & $2 \pm 0,4 \mathrm{~A}, \mathrm{a}$ & $2 \pm 0,7 \mathrm{~A}, \mathrm{a}$ & $1 \pm 0,3 \mathrm{~A}, \mathrm{bc}$ \\
\hline \multirow{4}{*}{$\frac{\mathrm{K}}{(\mathrm{mEq} / \mathrm{L})}$} & GI & $3 \pm 1 \mathrm{~A}, \mathrm{a}$ & $4 \pm 1 \mathrm{~A}, \mathrm{a}$ & $4 \pm 1 \mathrm{~A}, \mathrm{a}$ & $4 \pm 1 \mathrm{~A}, \mathrm{a}$ & $4 \pm 1 \mathrm{~A}, \mathrm{a}$ & $3 \pm 1 \mathrm{~A}, \mathrm{a}$ \\
\hline & GII & $4 \pm 1 \mathrm{~A}, \mathrm{a}$ & $4 \pm 1 \mathrm{~A}, \mathrm{a}$ & $3 \pm 0,2 \mathrm{~A}, \mathrm{a}$ & $4 \pm 1 \mathrm{~A}, \mathrm{a}$ & $4 \pm 1 \mathrm{~A}, \mathrm{a}$ & $4 \pm 1 \mathrm{~A}, \mathrm{a}$ \\
\hline & GIII & $4 \pm 0,3 \mathrm{~A}, \mathrm{a}$ & $3 \pm 0,3 \mathrm{~A}, \mathrm{a}$ & $5 \pm 5 \mathrm{~A}, \mathrm{a}$ & $4 \pm 1 \mathrm{~A}, \mathrm{a}$ & $4 \pm 1 \mathrm{~A}, \mathrm{a}$ & $3 \pm 0,4 \mathrm{~A}, \mathrm{a}$ \\
\hline & GIV & $3 \pm 1 \mathrm{~A}, \mathrm{a}$ & $3 \pm 0,4 \mathrm{~A}, \mathrm{a}$ & $3 \pm 0,2 \mathrm{~A}, \mathrm{a}$ & $3 \pm 1 \mathrm{~A}, \mathrm{a}$ & $4 \pm 1 \mathrm{~A}, \mathrm{a}$ & $3 \pm 0,4 \mathrm{~A}, \mathrm{a}$ \\
\hline \multirow{4}{*}{$\begin{array}{c}\mathrm{Na} \\
(\mathrm{mEq} / \mathrm{L})\end{array}$} & GI & $142 \pm 6 \mathrm{~A}, \mathrm{a}$ & $138 \pm 6 \mathrm{~A}, \mathrm{a}$ & $138 \pm 6 \mathrm{~A}, \mathrm{a}$ & $138 \pm 6 \mathrm{~A}, \mathrm{a}$ & $139 \pm 6 \mathrm{~A}, \mathrm{a}$ & $139 \pm 6 \mathrm{~A}, \mathrm{a}$ \\
\hline & GII & $136 \pm 5 \mathrm{~A}, \mathrm{a}$ & $136 \pm 6 \mathrm{~A}, \mathrm{a}$ & $138 \pm 5 \mathrm{~A}, \mathrm{a}$ & $135 \pm 5 \mathrm{~A}, \mathrm{a}$ & $137 \pm 6 \mathrm{~A}, \mathrm{a}$ & $140 \pm 8 \mathrm{~A}, \mathrm{a}$ \\
\hline & GIII & $140 \pm 3 \mathrm{~A}, \mathrm{a}$ & $139 \pm 3 \mathrm{~A}, \mathrm{a}$ & $137 \pm 5 \mathrm{~A}, \mathrm{a}$ & $138 \pm 3 \mathrm{~A}, \mathrm{a}$ & $140 \pm 6 \mathrm{~A}, \mathrm{a}$ & $140 \pm 2 \mathrm{~A}, \mathrm{a}$ \\
\hline & GIV & $139 \pm 5 \mathrm{~A}, \mathrm{ab}$ & $138 \pm 3 \mathrm{~A}, \mathrm{ab}$ & $137 \pm 3 \mathrm{~A}, \mathrm{ab}$ & $137 \pm 5 \mathrm{~A}, \mathrm{ab}$ & $135 \pm 3 \mathrm{~A}, \mathrm{~b}$ & $141 \pm 4 \mathrm{~A}, \mathrm{a}$ \\
\hline \multirow{4}{*}{$\begin{array}{c}\mathrm{Ca} \\
(\mathrm{mmol} / \mathrm{L})\end{array}$} & GI & $1 \pm 0,3 \mathrm{~A}, \mathrm{a}$ & $1 \pm 0,2 \mathrm{~A}, \mathrm{a}$ & $1 \pm 0,3 \mathrm{~A}, \mathrm{a}$ & $1 \pm 0,2 \mathrm{~A}, \mathrm{a}$ & $1 \pm 0,2 \mathrm{~A}, \mathrm{a}$ & $1 \pm 0,4 \mathrm{~A}, \mathrm{a}$ \\
\hline & GII & $1 \pm 0,2 \mathrm{~A}, \mathrm{a}$ & $1 \pm 0,2 \mathrm{~A}, \mathrm{a}$ & $1 \pm 0,2 \mathrm{~A}, \mathrm{a}$ & $1 \pm 0,2 \mathrm{~A}, \mathrm{a}$ & $1 \pm 0,3 \mathrm{~A}, \mathrm{a}$ & $1 \pm 0,5 \mathrm{~A}, \mathrm{a}$ \\
\hline & GIII & $1 \pm 0,2 \mathrm{~A}, \mathrm{a}$ & $1 \pm 0,2 \mathrm{~A}, \mathrm{a}$ & $1 \pm 0,1 \mathrm{~A}, \mathrm{a}$ & $1 \pm 0,1 \mathrm{~A}, \mathrm{a}$ & $1 \pm 0,4 \mathrm{~A}, \mathrm{a}$ & $1 \pm 0,2 \mathrm{~A}, \mathrm{a}$ \\
\hline & GIV & $1 \pm 0,2 \mathrm{~A}, \mathrm{a}$ & $1 \pm 0,2 \mathrm{~A}, \mathrm{a}$ & $1 \pm 0,2 \mathrm{~A}, \mathrm{a}$ & $1 \pm 0,2 \mathrm{~A}, \mathrm{a}$ & $1 \pm 0,1 \mathrm{~A}, \mathrm{a}$ & $1 \pm 0,2 \mathrm{~A}, \mathrm{a}$ \\
\hline
\end{tabular}


(...continuation)

\begin{tabular}{|c|c|c|c|c|c|c|c|}
\hline \multirow{4}{*}{$\begin{array}{c}\text { Glu } \\
(\mathrm{mg} / \mathrm{dl})\end{array}$} & GI & $73 \pm 13 \mathrm{~A}, \mathrm{~b}$ & $90 \pm 22 \mathrm{~A}, \mathrm{~b}$ & $115 \pm 22 \mathrm{~A}, \mathrm{ab}$ & $152 \pm 58 \mathrm{~A}, \mathrm{a}$ & $152 \pm 62 \mathrm{~A}, \mathrm{a}$ & $84 \pm 15 \mathrm{~A}, \mathrm{~b}$ \\
\hline & GII & $75 \pm 10 \mathrm{~A}, \mathrm{c}$ & $99 \pm 27$ A,bc & $138 \pm 33 \mathrm{~A}, \mathrm{ab}$ & $165 \pm 26 \mathrm{~A}, \mathrm{a}$ & $166 \pm 38 \mathrm{~A}, \mathrm{a}$ & $77 \pm 14 \mathrm{~A}, \mathrm{c}$ \\
\hline & GIII & $68 \pm 12 A, c$ & $100 \pm 22 \mathrm{~A}, \mathrm{abc}$ & $125 \pm 36 \mathrm{~A}, \mathrm{ab}$ & $139 \pm 50 \mathrm{~A}, \mathrm{a}$ & $125 \pm 40 \mathrm{~A}, \mathrm{ab}$ & $82 \pm 19$ A,bc \\
\hline & GIV & $67 \pm 8 \mathrm{~A}, \mathrm{c}$ & $101 \pm 17 \mathrm{~A}, \mathrm{bc}$ & $132 \pm 37 \mathrm{~A}, \mathrm{ab}$ & $153 \pm 49 \mathrm{~A}, \mathrm{a}$ & $139 \pm 47 \mathrm{~A}, \mathrm{ab}$ & $72 \pm 13 \mathrm{~A}, \mathrm{c}$ \\
\hline
\end{tabular}

Table 3. Biochemical values of groups (mean $\pm \mathrm{SD})$.

\begin{tabular}{ccccc}
\hline Value & Group & $0 \mathrm{~min}$ & $90 \mathrm{~min}$ & $24 \mathrm{~h}$ \\
\hline \multirow{3}{*}{ BUN $(\mathrm{mg} / \mathrm{dL})$} & GI & $45 \pm 1 \mathrm{~A}, \mathrm{a}$ & $49 \pm 8 \mathrm{AB}, \mathrm{a}$ & $45 \pm 10 \mathrm{~A}, \mathrm{a}$ \\
& GII & $41 \pm 5 \mathrm{~A}, \mathrm{a}$ & $40 \pm 3 \mathrm{~B}, \mathrm{a}$ & $41 \pm 3 \mathrm{~A}, \mathrm{a}$ \\
& GIII & $47 \pm 8 \mathrm{~A}, \mathrm{a}$ & $47 \pm 7 \mathrm{AB}, \mathrm{a}$ & $48 \pm 6 \mathrm{~A}, \mathrm{a}$ \\
& GIV & $47 \pm 10 \mathrm{~A}, \mathrm{a}$ & $50 \pm 9 \mathrm{~A}, \mathrm{a}$ & $47 \pm 9 \mathrm{~A}, \mathrm{a}$ \\
\hline GI & $1,6 \pm 1 \mathrm{~A}, \mathrm{a}$ & $2 \pm 1 \mathrm{~A}, \mathrm{a}$ & $1,6 \pm 1 \mathrm{~A}, \mathrm{a}$ \\
& GII & $1 \pm 1 \mathrm{~A}, \mathrm{a}$ & $1 \pm 1 \mathrm{~A}, \mathrm{a}$ & $1 \pm 1 \mathrm{~A}, \mathrm{a}$ \\
& GIII & $1 \pm 1 \mathrm{~A}, \mathrm{a}$ & $2 \pm 1 \mathrm{~A}, \mathrm{a}$ & $1 \pm 1 \mathrm{~A}, \mathrm{a}$ \\
& GIV & $1 \pm 1 \mathrm{~A}, \mathrm{a}$ & $1 \pm 1 \mathrm{~A}, \mathrm{a}$ & $1 \pm 1 \mathrm{~A}, \mathrm{a}$ \\
\hline & GI & $164 \pm 78 \mathrm{~A}, \mathrm{a}$ & $176 \pm 88 \mathrm{~A}, \mathrm{a}$ & $201 \pm 88 \mathrm{~A}, \mathrm{a}$ \\
& GII & $196 \pm 50 \mathrm{~A}, \mathrm{a}$ & $177 \pm 34 \mathrm{~A}, \mathrm{a}$ & $212 \pm 40 \mathrm{~A}, \mathrm{a}$ \\
& GIII & $200 \pm 30 \mathrm{~A}, \mathrm{a}$ & $177 \pm 29 \mathrm{~A}, \mathrm{a}$ & $205 \pm 18 \mathrm{~A}, \mathrm{a}$ \\
& GIV & $217 \pm 30 \mathrm{~A}, \mathrm{a}$ & $204 \pm 31 \mathrm{~A}, \mathrm{a}$ & $232 \pm 80 \mathrm{~A}, \mathrm{a}$ \\
\hline GLP $(\mathrm{IU} / \mathrm{L})$ & GI & $222 \pm 39 \mathrm{~A}, \mathrm{a}$ & $228 \pm 46 \mathrm{~A}, \mathrm{a}$ & $231 \pm 54 \mathrm{~A}, \mathrm{a}$ \\
& GII & $210 \pm 33 \mathrm{~A}, \mathrm{a}$ & $184 \pm 66 \mathrm{~A}, \mathrm{a}$ & $221 \pm 40 \mathrm{~A}, \mathrm{a}$ \\
& GIII & $195 \pm 18 \mathrm{~A}, \mathrm{a}$ & $174 \pm 40 \mathrm{~A}, \mathrm{a}$ & $205 \pm 26 \mathrm{~A}, \mathrm{a}$ \\
& GIV & $184 \pm 19 \mathrm{~A}, \mathrm{a}$ & $158 \pm 37 \mathrm{~A}, \mathrm{a}$ & $159 \pm 74 \mathrm{~A}, \mathrm{a}$ \\
\hline
\end{tabular}

BUN: blood urea nitrogen, Cr: Creatine, AST: Aspartate Transaminase, ALP: Alkaline Phosphatase, Ck-MB: Creatine kinase $\mathrm{MB}, \mathrm{A}, \mathrm{B}, \mathrm{C}$ in the same column and a, b, c in the same row are statistically significant $(P<0.05)$.

\section{RESULTS}

Appropriate and adequate sedation was observed in all of the animals following the induction of anesthesia. No significant differences were observed in the times for loss of palpebral reflex, extubation, sternoabdominal position and standing up times (Table 1). A difference was seen in the time for return of palpebral reflex between groups I and III $(P<0.05)$; it was shorter in group III than in group I. Atipamezole did not affect these times in group II or IV.

Hematological parameters, blood gas levels, and electrolyte levels were measured before, during, at the end of, and $24 \mathrm{~h}$ after anesthesia; these values are shown in Table 2. There were significant differ- ences found in WBC and $\mathrm{Hb}$ values within groups and between groups, but these differences were in reference interval. The blood $\mathrm{pH}$ increased in all groups during anesthesia, and significant differences were detected $(P<0.05)$. However, the $\mathrm{pH}$ returned to values within the normal range $24 \mathrm{~h}$ after anesthesia. $\mathrm{PCO}_{2}, \mathrm{HCO}_{-3}$, and $\mathrm{tCO}_{2}$ levels decreased between $15 \mathrm{~min}$ and $90 \mathrm{~min}$ after induction of anesthesia. The decreases in these values were statistically significant in all groups $(P<$ 0.05). The Lac levels increased between $15 \mathrm{~min}$ and 90 min after induction of anesthesia in all groups. The increases in these values were statistically significant in all groups $(P<0.05)$. $\mathrm{PO}_{2}$ levels were different among groups I, II, and IV $15 \mathrm{~min}$ after induction of 
anesthesia $(P<0.05)$. The highest level of $\mathrm{PO}_{2}$ was recorded in group IV. $\mathrm{Na}, \mathrm{K}$, and Ca levels were within the reference intervals during anesthesia and $24 \mathrm{~h}$ after anesthesia. Glu levels increased between $15 \mathrm{~min}$ and 90 min after induction of anesthesia in all groups, and the increase was statistically significant $(P<0.05)$.

Biochemical parameters were evaluated before, at the end of, and $24 \mathrm{~h}$ after anesthesia (Table 3). At $90 \mathrm{~min}$ after induction of anesthesia, the only difference observed was in the levels of BUN between groups II and IV $(P<0.05)$. No statistically significant differences in Cr, ALT, AST, and Ck-MB levels were observed within or between groups.

Regarding cardiopulmonary findings, no significant differences were seen in heart rate or $\mathrm{SPO}_{2}$ levels between and among groups. Decreases in respiratory rates and body temperatures were detected in all groups (Table 4), and these decreases were statistically significant $(P<0.05)$. The comparison of systolic, mean, and diastolic pressures significant differences were seen between and among groups. Blood pressure decreased between $15 \mathrm{~min}$ and $90 \mathrm{~min}$ during anesthesia.

Sinus bradycardia and increased $\mathrm{T}$ waves were observed in ECG tracings, but no atrioventricular blocks were detected.

There were no significant differences observed in $\mathrm{ETCO}_{2}$ levels between groups, but intra-group evaluation in groups II, III, and IV showed significant differences at $0 \mathrm{~min}$ and 15, 30, 60, $90 \mathrm{~min}$ (Table 5).

Table 4. Cardiopulmonary values of groups (mean $\pm \mathrm{SD}$ ).

\begin{tabular}{cccccccc}
\hline Value & Group & $0 \mathrm{~min}$ & $15 \mathrm{~min}$ & $30 \mathrm{~min}$ & $60 \mathrm{~min}$ & $90 \mathrm{~min}$ & $24 \mathrm{~h}$ \\
\hline & GI & $146 \pm 74 \mathrm{~A}, \mathrm{a}$ & $143 \pm 31 \mathrm{~A}, \mathrm{a}$ & $117 \pm 38 \mathrm{~A}, \mathrm{a}$ & $124 \pm 18 \mathrm{~A}, \mathrm{a}$ & $96 \pm 27 \mathrm{~A}, \mathrm{a}$ & $145 \pm 70 \mathrm{~A}, \mathrm{a}$ \\
$\mathrm{SP}$ & GII & $148 \pm 53 \mathrm{~A}, \mathrm{a}$ & $163 \pm 53 \mathrm{~A}, \mathrm{a}$ & $115 \pm 36 \mathrm{~A}, \mathrm{a}$ & $115 \pm 41 \mathrm{~A}, \mathrm{a}$ & $125 \pm 41 \mathrm{~A}, \mathrm{a}$ & $150 \pm 48 \mathrm{~A}, \mathrm{a}$ \\
& GIII & $181 \pm 49 \mathrm{~A}, \mathrm{a}$ & $138 \pm 35 \mathrm{~A}, \mathrm{abc}$ & $107 \pm 20 \mathrm{~A}, \mathrm{bc}$ & $98 \pm 24 \mathrm{~A}, \mathrm{c}$ & $102 \pm 22 \mathrm{~A}, \mathrm{bc}$ & $153 \pm 52 \mathrm{~A}, \mathrm{ab}$ \\
& GIV & $190 \pm 55 \mathrm{~A}, \mathrm{a}$ & $122 \pm 23 \mathrm{~A}, \mathrm{bc}$ & $90 \pm 17 \mathrm{~A}, \mathrm{c}$ & $101 \pm 18 \mathrm{~A}, \mathrm{c}$ & $92 \pm 12 \mathrm{~A}, \mathrm{c}$ & $174 \pm 59 \mathrm{~A}, \mathrm{ab}$ \\
\hline & GI & $100 \pm 59 \mathrm{~A}, \mathrm{a}$ & $84 \pm 22 \mathrm{~A}, \mathrm{a}$ & $73 \pm 27 \mathrm{~A}, \mathrm{a}$ & $79 \pm 19 \mathrm{~A}, \mathrm{a}$ & $59 \pm 27 \mathrm{AB}, \mathrm{a}$ & $93 \pm 64 \mathrm{~A}, \mathrm{a}$ \\
& GII & $88 \pm 69 \mathrm{~A}, \mathrm{a}$ & $119 \pm 70 \mathrm{~A}, \mathrm{a}$ & $80 \pm 42 \mathrm{~A}, \mathrm{a}$ & $70 \pm 40 \mathrm{AB}, \mathrm{a}$ & $81 \pm 42 \mathrm{~A}, \mathrm{a}$ & $106 \pm 41 \mathrm{~A}, \mathrm{a}$ \\
$\mathrm{DP}$ & GIII & $130 \pm 49 \mathrm{~A}, \mathrm{a}$ & $90 \pm 25 \mathrm{~A}, \mathrm{abc}$ & $60 \pm 24 \mathrm{~A}, \mathrm{bc}$ & $47 \pm 17 \mathrm{AB}, \mathrm{c}$ & $52 \pm 13 \mathrm{AB}, \mathrm{c}$ & $107 \pm 50 \mathrm{~A}, \mathrm{ab}$ \\
& GIV & $130 \pm 60 \mathrm{~A}, \mathrm{a}$ & $75 \pm 22 \mathrm{~A}, \mathrm{bc}$ & $51 \pm 16 \mathrm{~A}, \mathrm{c}$ & $46 \pm 10 \mathrm{~B}, \mathrm{c}$ & $44 \pm 12 \mathrm{~B}, \mathrm{c}$ & $106 \pm 44 \mathrm{~A}, \mathrm{ab}$ \\
\hline & GI & $116 \pm 69 \mathrm{~A}, \mathrm{a}$ & $118 \pm 34 \mathrm{~A}, \mathrm{a}$ & $90 \pm 32 \mathrm{~A}, \mathrm{a}$ & $88 \pm 13 \mathrm{~A}, \mathrm{a}$ & $74 \pm 18 \mathrm{AB}, \mathrm{a}$ & $120 \pm 72 \mathrm{~A}, \mathrm{a}$ \\
$\mathrm{MP}$ & GII & $105 \pm 69 \mathrm{~A}, \mathrm{a}$ & $119 \pm 59 \mathrm{~A}, \mathrm{a}$ & $88 \pm 31 \mathrm{~A}, \mathrm{a}$ & $87 \pm 47 \mathrm{~A}, \mathrm{a}$ & $98 \pm 47 \mathrm{~A}, \mathrm{a}$ & $111 \pm 51 \mathrm{~A}, \mathrm{a}$ \\
& GIII & $143 \pm 59 \mathrm{~A}, \mathrm{a}$ & $107 \pm 34 \mathrm{~A}, \mathrm{abc}$ & $77 \pm 23 \mathrm{~A}, \mathrm{bc}$ & $64 \pm 27 \mathrm{~A}, \mathrm{c}$ & $67 \pm 15 \mathrm{AB}, \mathrm{bc}$ & $125 \pm 58 \mathrm{~A}, \mathrm{ab}$ \\
& GIV & $150 \pm 55 \mathrm{~A}, \mathrm{a}$ & $99 \pm 25 \mathrm{~A}, \mathrm{bc}$ & $67 \pm 20 \mathrm{~A}, \mathrm{c}$ & $64 \pm 15 \mathrm{~A}, \mathrm{c}$ & $58 \pm 14 \mathrm{~B}, \mathrm{c}$ & $125 \pm 47 \mathrm{~A}, \mathrm{ab}$ \\
\hline
\end{tabular}

SP: Sistolic Pressure, DP: Diastolic Pressure, MP: Mean pressure, A, B, C in the same column and a, b, c in the same row are statistically significant $(P<0.05)$.

Table 5. $\mathrm{ETCO}_{2}$ values of groups (mean $\left.\pm \mathrm{SD}\right)$.

\begin{tabular}{ccccccc}
\hline Value & Group & $0 \mathrm{~min}$ & $15 \mathrm{~min}$ & $30 \mathrm{~min}$ & $60 \mathrm{~min}$ & $90 \mathrm{~min}$ \\
\hline & GI & $41 \pm 11 \mathrm{~A}, \mathrm{a}$ & $37 \pm 9 \mathrm{~A}, \mathrm{a}$ & $34 \pm 10 \mathrm{~A}, \mathrm{a}$ & $33 \pm 8 \mathrm{~A}, \mathrm{a}$ & $31 \pm 10 \mathrm{~A}, \mathrm{a}$ \\
$\mathrm{ETCO}_{2}$ & GII & $41 \pm 8 \mathrm{~A}, \mathrm{a}$ & $36 \pm 8 \mathrm{~A}, \mathrm{ab}$ & $31 \pm 9 \mathrm{~A}, \mathrm{ab}$ & $29 \pm 10 \mathrm{~A}, \mathrm{~b}$ & $29 \pm 9 \mathrm{~A}, \mathrm{~b}$ \\
& GIII & $50 \pm 9 \mathrm{~A}, \mathrm{a}$ & $38 \pm 4 \mathrm{~A}, \mathrm{~b}$ & $35 \pm 3 \mathrm{~A}, \mathrm{~b}$ & $33 \pm 3 \mathrm{~A}, \mathrm{~b}$ & $33 \pm 3 \mathrm{~A}, \mathrm{~b}$ \\
& GIV & $50 \pm 10 \mathrm{~A}, \mathrm{a}$ & $38 \pm 7 \mathrm{~A}, \mathrm{~b}$ & $33 \pm 8 \mathrm{~A}, \mathrm{~b}$ & $30 \pm 6 \mathrm{~A}, \mathrm{~b}$ & $28 \pm 6 \mathrm{~A}, \mathrm{~b}$ \\
\hline
\end{tabular}

\section{DISCUSSION}

Numerous preanesthetic and inhaled anesthetic agents are used in horses, including phenothiazines, $a-2$ agonists, opioids, isoflurane, and sevoflurane $[1,20]$. Before administering anesthesic agents in horses, clinical and laboratory examinations should be performed [8,9]. In previous studies [8,28] $10 \mu \mathrm{g} /$ $\mathrm{kg}$ medetomidine was administered intravenously in horses as premedication, achieving heavy sedation and shorter time for enter preanesthesia. Arican et al. [1] intravenously administered $7 \mu \mathrm{g} / \mathrm{kg}$ medetomidine in horses, and adequate sedation was obtained. In this study, medetomidine $(7 \mu \mathrm{g} / \mathrm{kg})$ and detomidine $(25$ $\mu \mathrm{g} / \mathrm{kg}$ ) were intravenously administered, which was adequate and suitable for sedating horses.

The times for return of palpebral reflex were statistically different in groups I and III; the time was shorter in group III than in group I. This result shows 
that the duration of action of detomidine is longer than that of medetomidine. At the end of anesthesia, 0.06 $\mathrm{mg} / \mathrm{kg}$ atipamezole was intravenously administered in groups II and IV. However, atipamezole did not affect the clinical parameters of times of palpebral reflex return, sternoabdominal position and standing up times. Concetto et al. [4] emphasized that the atipamezole dose should be 5-10 times higher than the dose of an $a-2$ agonist in horses. Preanesthetic and anesthetic agents suppress respiration, lower the heart rate, and decrease organ perfusion. Thus in groups II and IV atipamezole exhibited prolonged binding to organ receptors, and did not shorten clinical parameter times.

Stress, excitement, fear, catecholamine exchange in blood circulation, hyperglycemia, and hypoxia can all cause changes in venous blood parameters [19]. These are potential reasons for the changes in venous blood parameters (i.e., WBC and $\mathrm{Hb}$ ) observed at the beginning of and during anesthesia in the present study.

The increases in venous blood $\mathrm{pH}$ values in all groups were caused by decreased levels of $\mathrm{PO}_{2}$. This condition was thought to be due to desflurane's low blood solubility coefficient and rapid alveolar accumulation, as well as its tendency to increase heart rate and its action as a respiratory depressant. When the $\mathrm{pH}$ changed, a decrease in $\mathrm{PCO}_{2}$ levels and an increase in $\mathrm{PO}_{2}$ levels were observed in all groups. Furthermore, it was concluded that the statistical differences of $\mathrm{HCO}_{3}$ and $\mathrm{tCO}_{2}$ between and among groups were occurred to counteract respiratory alkalosis.

Alpha 2-agonists and ketamine-midazolam anesthetic combinations decrease $\mathrm{PO}_{2}$ levels and increase $\mathrm{PCO}_{2}$ levels in horses [30]. The opposite occurred after administration of our anesthetic combinations. This was because general anesthesia with oxygen supplementation and positive pressure on the thorax slowed venous circulation, decreased cardiac output, and alteration of $\mathrm{PCO}_{2}$ levels. After $24 \mathrm{~h}$, the effects of the anesthetic agents wore off, and blood gas, enyzme and ECG values returned to normal values. In ventilation assisted general anesthesia, circulating depression not cause to major problem in healty horses [12]. In our study, the horses had normal electrolyte levels and blood volume, and no major problems were seen. This is in accordance with the results of published studies. In animals under general anesthesia, anaerobic metabolism begins to produce lactate because of decreased tissue perfusion; this increases the amount of lactate in the body during anesthesia.

Even though sympathetic stimulation and lipolysis increase during general anesthesia in healthy animals, lactate produced by anaerobic metabolism in the muscles is balanced by decreasing adenosine triphosphate and creatine phosphokinase [24]. Detected changes in lactate levels in this study are dependent on this cause.

During and after the anesthetic period, serum biochemical values can be different from baseline values. They are dependent on the effects of anesthetic agents. During anesthesia, the decrease and increase of biochemical values stabilize the changes in the enzyme system that develops because of the effects of anesthetic agents $[10,23]$. In the present study, it was considered that the changes in the biochemical values aimed to stabilize the changes induced by anesthesia.

It was reported that desflurane causes vasodilation in subcutaneous tissues, changes in heart rate and respiration, and decreased body temperature [17,21]. The decrease in body temperature of the horses was usually dependent on skin thickness and ambient temperature 25]. The information in the literature $[21,25]$ explained the changes in body temperature observed in our study.

The heart rate may be high before anesthesia in healthy horses because of psychological conditions; therefore, the values of the ECG results (heart rate, systolic pressure, mean pressure, diastolic pressure) may be higher than normal [15]. In our study, systolic pressure before anesthesia was observed to be higher than normal in all groups. This showed that the changes in heart rate during anesthesia could be due to psychological factors. Regarding the electrolyte parameters evaluated in the study, there was a statistical difference detected in $\mathrm{Na}$ values between 90 min after induction of anesthesia and $24 \mathrm{~h}$ after induction of anesthesia in group IV. However, in previous studies, the changes in $\mathrm{Na}$ values did not influence the cardiac pressure during general anesthesia. In our study, significant changes were not seen in any electrolyte parameters except $\mathrm{Na}$, and atrioventricular block was not detected in ECG traces.

Desflurane anesthesia decreases pulmonary tidal volume at all MAC values. Desflurane (1 and 1.75 MAC) decreases respiratory rate but does not affect heart rate or mean arterial pressure [21,26,27]. 
In the current study, 2 MAC desflurane was initially administered, followed by a decrease to 1 MAC after 30 min. The obtained data showed decreased $\mathrm{PCO}_{2}$ levels and increased $\mathrm{PO}_{2}$ levels. These differences are thought to be due to the use of preanesthetics, ketamine-midazolam, and varying desflurane doses.

Generally, decreased $\mathrm{ETCO}_{2}$ levels are evidence of lung perfusion deficiency. It depends on the effects of anesthetic agents on the cardiopulmonary, cardiovascular, and respiratory systems. In particular, the higher pressure and dose of desflurane supress respiratory system. Oxygen supplementation in general anesthesia increases respiratory rate, but a- 2 agonists and ketamine-midazolam effects can eliminate the increasing respiratory rate in general anesthesia.

\section{CONCLUSION}

The anesthetic procedures and combinations used in this study are safe and suitable for use in horses. Desflurane is an ideal volatile anesthetic agent for horses. The results of this study suggest that desflurane can be used in patients with cardiovascular, liver, and urinary system diseases. However, caution must be exercised when the MAC value is exceeded, as it can cause negative effects on the cardiovascular system due to faster gas alteration of alveolar concentration.

\section{MANUFACTURERS}

${ }^{1}$ Bionet Co, Ldt. Istanbul, Turkey.

${ }^{2}$ MedAir AB. Hudiksvall, Sweden.

${ }^{3}$ Pfizer Ilaçları Ldt. Sti. Istanbul, Turkey.

${ }^{4}$ Bremer Pharma Gmbh. Bremerhaven, Germany.

${ }^{5}$ Curamed Pharma GmbH. Karlsruhe, Germany.

${ }^{6}$ SurgiVet (Smith Medical). Minneapolis, MN, USA.

${ }^{7}$ Eczacıbaşı - Baxter. Istanbul, Turkey.

${ }^{8}$ Instrumentation Laboratory Inc. Bedford, MA, USA.

${ }^{9}$ Melet Schlosing Laboratories. Osny, France.

Acknowledgements. This study was supportted by Selcuk University Scientific Research Projects (Project No: BAP 2010/10102020).

Ethical approval. The present study was performed in the Department of Surgery, Faculty of Veterinary Medicine, University of Selcuk, Turkey and was approved by the Ethics Committee of Veterinary Medicine (Numbered: 2010/027).

Declaration of interest. The authors report no conflicts of interest. The authors alone are responsible for the content and writing of the paper.

\section{REFERENCES}

1 Arican M., Erol H. \& Esin E. 2015. Clinical comparison of medetomidine with isoflurane or sevoflurane for anesthesia in horses. Pakistan Veterinary Journal. 35(4): 474-478.

2 Bettschart-Wolfensberger R., Bowen I.M., Freeman S.L., Weller R. \& Clarke K.W. 2003. Medetomidine-ketamine anaesthesia induction followed by medetomidine-propofol in ponies:infusion rates and cardiopulmonary side effects. Equine Veterinary Journal. 35(3): 308-313.

3 But K.A., Durmus M., Toprak H.I., Ozturk E., Demirbilek S. \& Ersoy M.O. 2005. Hemodynamic, hepatorenal, and postoperative effects of desflurane-fentanly and midazolam-fentanly anesthesia in coronary artery bypass surgery. Journal of Cardiothoracic and Vascular Anesthesia. 19(5): 597-602.

4 Concetto S.D., Archer R.M., Sigurdsson S.F. \& Clarke K.W. 2007. Atipamezole in the management of detomidine overdose in a pony. Veterinary Anaesthesia and Analgesia. 34: 67-69.

5 Dugdale A. 2010. Veterinary anaesthesia principles to practice. Oxford: Blackwell Publishing Ltd., pp.68-100.

6 Elfenbein J.R., Sanchez L.C., Robertson S.A., Cole C.A. \& Sams R. 2009. Effect of detomidine on visceral and somatic nociception and deudonal motility in conscious adult horses. Veterinary Anaesthesia and Analgesia. 36: 162-172.

7 Giovannoni M.P., Gbelardani C., Vergelli C. \& Piaz V.D. 2009. a2-Agonists as analgesic agents. International Journal of Medical Research Review. 29(2): 339-368.

8 Grimsud K.N., Ait-Oudhia S., Durbin Johnson B.P., Rocke D.M., Mama K.R. Rezende M.L., Stanley S.D. \& Jusko W.J. 2015. Pharmacokinetic and pharmacodynamic analysis comparing diverse effects of detomidine, medetomidine, and dexmedetomidine in the horse: a population analysis. Journal of Veterinary Pharmacology and Therapeutics. 38(1): 24-34.

9 Hall L.W., Clarke K.W. \& Trim C.M. 2000. Veterinary Anaesthesia. 10th edn. London: W.B. Saunders, pp.75-112 \& 247-313.

10 Hubbell J.A.E., Aaernes T.K., Bednarski R.M., Lerche P. \& Muir W.M. 2011. Effects of $50 \%$ and maximal inspired oxygen concentrations on respiratory variables in isoflurane-anesthetized horses. BMC Veterinary Research. 7 (23): 1-11. 
11 Ko J.C.H., Fox S.M. \& Mandsager R.E. 2000. Sedative and cardiorespiratory effects of medetomidine, medetomidinebutarphanol, and medetomidine- ketamine in dogs. Journal of the American Veterinary Medical Association. 216: 1578-1583.

12 Kushiro T., Yamashita K., Umar M.A., Maehara S., Wakaiki S., Abe R., Seno T., Tsuzuki K., Izumisawa Y. \& Muir W.M. 2005. Anesthesic and cardiovascular effects of balanced anesthesia using constant rate infusion of midazolam-ketamine-medetomidine with inhalation of oxygen-sevoflurane (MKM-OS Anesthesia) in horses. The Journal of Veterinary Medical Science. 67(4): 379-384.

13 Levionnois O.L., Menge M., Thormann W., Mevissen M. \& Spadavecchia C. 2010. Effect of ketamine on the limb withdrawal reflex evoked by transcutaneous electrical stimulation in ponies anaesthetised with isoflurane. The Veterinary Journal. 186(3): 304-311.

14 Massoco C. \& Palermo-Neto J. 2003. Effects of midazolam on equine innate immune response: a flow cytometric study. Veterinary Immunology and Immunopathology. 95: 11-19.

15 Morgan R.A., Raftery A.G., Cripps P., Senior J.M. \& McGowan C.M. 2011. The prevalence and nature of cardiac arrhythmias in horses following general anaesthesia and surgery. Acta Veterinaria Scandinavica. 53(62): 1-33.

16 Natalini C.C. 2001. Sevoflurane, desflurane and xenon new inhaled anesthetics in veterinary medicine. Ciência Rural. 31(1): 177-183.

17 Oku K., Kazıkazı M., Ono K. \& Ohta M. 2011. Clinical evaluation of total intravenous anesthesia using a combination of propofol and medetomidine following anesthesia induction with medetomidine, guaifenesin and propofol for castration in thoroughbred horses. The Journal of Veterinary Medical Science. 73(12): 1639-1643.

18 Pypendorp B., Serteny D. \& Verstegen J. 1996. Hemodynamic effects of medetomidine-midazolam butorphanol and medetomidine-midazolam-buprenorphine combination and reversibility by atipamezole in dogs. American Journal of Veterinary Research. 57: 724-730.

19 Robinson N.E. 2009. The respiratory system. In: Muir W. \& Hubbell J.A.E. (Eds). Equine anaesthesia: monitoring and emergency therapy. 2nd edn. Saint Louis: Saunders Elsevier, pp.11-34.

20 Rosetti R.B., Cortopassi S.R.G., Intelizano T., Machado T.S.L. \& Da Cruz R.S.F. 2008. Comparision of ketamine and $\mathrm{S}(+)$-ketamine, with romifidine and diazepam, for total intravenous anesthesia in horses. Veterinary Anaesthesia and Analgesia. 35: 30-37.

21 Santos M., Lopez-Sanroman J., Garcia-Iturralde P., Fuente M. \& Tendillo F.J. 2005. Cardiopulmoner effects of desflurane in horses. Veterinary Anesthesia and Analgesia. 32: 355-359.

22 Steffey E.P. 2002. Detomidine reduces isoflurane anesthetic requirement (MAC) in horses. Veterinary Anesthesia and Analgesia. 29: 223-27.

23 Steffey E.P. 2009. Inhalation anesthetics and gases. In: Muir W. \& Hubbell J.A.E. (Eds). Equine anaesthesia: monitoring and emergency therapy. 2nd edn. Saint Louis: Saunders Elsevier, pp.288-314.

24 Taylor P.M. 1998. Effects of hypercapnia on endocrine and metabolic responses to anaesthesia in ponies. Research in Veterinary Science. 65: 41-46.

25 Taylor P.M. \& Clerk K.W. 2007. Handbook of equine anaesthesia. 2nd edn. Philadelphia: Saunders Elsevier, pp.1-30.

26 Tendillo F.J., Mascias A., Santos M., Lopez-Sanroman J., Rossi R.D., Roman F.S. \& Segura I.A.G. 1997. Anesthetic potency of desflurane in the horse: determination of the minimum alveoler concentration. Veterinary Surgery. 26: 354-357.

27 Villamandos R.J.G., Palacios C., Benitez A., Granados M.M., Dominguez J.M., Estapa J.C., Ruız I., Aguilera E. \& Santisteban J.M. 2008. Effect of medetomidine infusion on the anaesthetic requirements of desflurane in dogs. Research in Veterinary Science. 84: 68-73.

28 Yamashita K., Muir W.W., Tsubakishita S., Abrahamsen E., Lerch P., Hubbell J.A.E., Bednarski R.M., Skarda R.T., Izumisawa Y. \& Kotani T. 2002. Clinical comparison of xylazine and medetomidine for premedication of horses. Journal of the American Veterinary Medical Association. 221(8): 1144-1149.

29 Yamashita K., Tsubakishita S., Futaoka S., Ueda I., Hamaguci H., Seno T., Katoh S., Uzimasawa Y. \& Muir W.W. 2000. Cardiovascular effects of medetomidine, detomidine and xylazine in horses. The Journal of Veterinary Medical Science. 62(10): 1025-1032.

30 Yamashita K., Wijayathilaka T.P., Kushiro T., Umar M.A., Taguchi K. \& Muir W.W. 2006. Anesthetic and cardiopulmonary effects of total intravenous anesthesia using a midazolam, ketamine and medetomidine drug combination in horses. The Journal of Veterinary Medical Science. 69(1): 7-13. 